\title{
PROSTATECTOMÍA RADICAL LAPAROSCÓPICA
}

\section{Primera intervención en Colombia}

Hernán Alonso Aponte Varón MD*, María del Pilar Ceballos MD**, René Sotelo MD***, Carlos Alberto Larios MD****

\section{Resumen}

A nivel mundial, la primera publicación de un procedimiento laparoscópico mayor en urología fue realizada por Ralph Claymann en 1991 y se trató de una nefrectomía. Tres años después, en julio de 1993, se practica el mismo procedimiento en el Hospital de San José. La primera prostatectomía radical laparoscópica fue descrita en 1992 por Schuessler y colaboradores, quienes en 1997 reportaron una serie de nueve casos sin demostrar beneficios superiores a los del procedimiento convencional. Fue en 1999, cuando Guillonneau y Vallancien informaron una serie de 65 pacientes donde describieron la técnica y demostraron la baja morbilidad, ofreciendo una alternativa quirúrgica a los pacientes con cáncer de próstata.

Se presenta el primer caso de prostatectomía radical laparoscópica realizada en Colombia por el Servicio de Urología del Hospital de San José, en un paciente de 60 años con un adenocarcinoma de próstata T2bNxM0; el tiempo quirúrgico fue de cinco horas, sin transfusión y dado de alta en el primer día postoperatorio.

Son muchas las ventajas de la cirugía laparoscópica en relación con la abierta, como disminución del sangrado por la excelente visibilidad del complejo dorsal y al efecto de taponamiento que realiza la presión del neumoperitoneo durante la cirugía.

Los índices de continencia y de estrechez de la anastomosis son comparables en las diferentes series abiertas y laparoscópicas, así como la tasa de márgenes positivos. De la misma forma, al tener una magnificación de las bandeletas neurovasculares, hay una mejor preservación de las mismas con buena respuesta eréctil posterior.

En conclusión, la prostatectomía radical laparoscópica es un procedimiento novedoso, con mejor preservación de las bandeletas nerviosas y menor sangrado, disminución de la estancia hospitalaria y recuperación más rápida, con menos requerimiento de analgésicos, y así mismo, una reincorporación laboral temprana.

\section{Introducción}

El Hospital de San José se ha caracterizado a través de la historia por estar a la vanguardia de la medicina en Colombia y en el campo de la urología no ha sido diferente. No es de extrañarse que el padre de la urología colombiana, Zoilo Cuéllar, haya sido uno de los fundadores de la Sociedad de Cirugía y del Hospital de San José.

La primera nefrectomía laparoscópica fue realizada por Ralph Claymann en 1990 y en julio de 1993 Raúl Parra, con la colaboración de Luis Blanco y Hernán Aponte, efectuaron la primera en Colombia,

\footnotetext{
* Jefe del Servicio de Urología, Hospital de San José.

** Residente III Urología, Hospital de San José.

*** Profesor Asistente, Fundación Universitaria de Ciencias de la Salud.

***** Urólogo Adscrito, Hospital de San José.
}

en una niña de 6 años con un riñón hipotrófico. Sin embargo, nos contagiamos del escepticismo que la comunidad urológica internacional vivió como consecuencia del poco beneficio inicial demostrado por el procedimiento, en comparación con la cirugía, por el alto costo y el poco desarrollo tecnológico que se presentaba en ese momento.

Aunque la adrenalectomía y la nefrectomía laparoscópicas podrían ser calificadas como el patrón de oro en muchas instituciones, hoy en día otros procedimientos laparoscópicos han caído en desuso, como la varicocelectomía laparoscópica, por sus altos costos y pocos beneficios en comparación con la cirugía abierta, y la cistopexia laparoscópica, por sus altos índices de fracasos y el advenimiento de técnicas mínimamente invasoras como la hamaca pubovaginal. 
Por otra parte, procedimientos urológicos mayores como la prostatectomía radical, que llevan poco tiempo de desarrollo, han entrado en competencia con las cirugías abiertas, por ser el procedimiento de elección. El debate a nivel mundial está abierto para el desarrollo de nuevas técnicas.

La prostatectomía radical laparoscópica fue descrita en 1992 por Schuessler y colaboradores. En 1997 publicaron una serie de nueve casos sin demostrar beneficios para el paciente. En 1999 Guillonneau y Vallancien reportaron una serie de 65 pacientes, donde describieron la técnica y demostraron la baja morbilidad, que presentaron como una alternativa quirúrgica para los pacientes con cáncer de próstata.

\section{Materiales y métodos}

Paciente de 60 años a quien en cita de control urológico se le encuentra al tacto rectal un aumento difuso de la consistencia y un nódulo en el lóbulo izquierdo, con recesos libres. El antígeno prostático específico (PSA) es de 31,9 por lo cual se le solicita biopsia transrectal de próstata, realizada en agosto de 2002 con diagnóstico de adenocarcinoma Gleason 3+3 grado 6, clasificado como T2BNXM0. El paciente tenía adecuada continencia urinaria y presentaba erecciones de baja calidad, sin actividad sexual. Se realiza gammagrafía ósea, que es negativa para metástasis, por lo cual se programa para prostatectomía radical laparoscópica.

Se hizo preparación de colon según el protocolo vigente en nuestro servicio para estos casos. Se administró anestesia general inhalatoria con el paciente en posición decúbito supino, con abducción de 45 grados de miembros inferiores, miembros superiores en aducción y posición de Trendelenburg.

El neumoperitoneo con $\mathrm{CO}_{2}$ se mantuvo a presión de $15 \mathrm{~mm} / \mathrm{Hg}$. El primer trócar de $10 \mathrm{~mm}$ se introdujo por una incisión vertical en la cicatriz umbilical, por donde se introdujo la cámara. El segundo trócar de $5 \mathrm{~mm}$ se colocó de 1 a $2 \mathrm{~cm}$ adentro y por encima de la espina iliaca anterosuperior izquierda. El siguiente de $5 \mathrm{~mm}$ en la línea pararrectal izquierda 1 a $2 \mathrm{~cm}$ por debajo de una línea imaginaria que une al ombligo con la espina anterosuperior izquierda. Los dos restantes de 5 y 10 mm se introdujeron en la ubicación contra-lateral a los trócares dos y tres.

Previa colocación de sonda de Foley 18fr se llenó la vejiga con 300 cc de solución salina normal, se practica disección y apertura del espacio prevesical, realizándose ingreso a través del peritoneo por una incisión de 2 a $3 \mathrm{~cm}$ por fuera de la circunferencia vesical, comenzando en la región lateral hacia la línea media, seccionando el uraco hasta los conductos deferentes. Esta disección llega hasta las ramas isquiopúbicas y el pubis mismo. Después se vacía el globo vesical y se incide la fascia endopélvica a cada lado, se cauteriza con bisturí armónico y se seccionan las venas superficiales del complejo dorsal, el cual se liga con una aguja CT1 de Vicryl 2-0 con doble lazada. Sobre la cara anterior de la próstata se liga el complejo proximal, lo cual permite la tracción de la próstata hacia la pared abdominal en el momento de disecar la unión vesicoprostática; la unión del cuello de la vejiga y la próstata se identifica con un beniqué curvo y con el asa en L del electrocauterio y el bisturí armónico, se realiza el corte del cuello de la vejiga tanto en la pared anterior como la posterior, hasta llegar a las vesículas seminales. Se practica disección y sección de los conductos deferentes y vesículas seminales, los cuales son levantados para proceder a hacer la apertura de la fascia de Denonvilliers. A los lados se evidencian los pedículos prostáticos, los cuales se seccionan con la tijera curva del bisturí armónico y se rodea la próstata hasta la base del ápex. En este caso no se preservaron las bandeletas neurovasculares debido a la extensión tumoral. Posteriormente se coagula y se secciona el complejo dorsal hasta la uretra y con la ayuda del beniqué se corta con tijera, primero la pared anterior y las laterales de la uretra y por último la cara posterior y el ligamento rectouretral, aislando la pieza operatoria, la cual se introduce en una bolsa de extracción. Se realiza la anastomosis uretrovesical con sutura polisorb 2-0 de aguja GU-46 5/8 punta atraumática de $25 \mathrm{~mm}$ de longitud, cuatro puntos a las $5,7,1$ y 11 , colocándose una sonda Foley 18 fr durante la misma. Se cierra en raqueta el cuello vesical restante. Se deja dren de látex por uno de los puertos cercano a la espina iliaca y se extrae la pieza por la cicatriz umbilical. 


\section{Resultados}

El tiempo quirúrgico fue de cinco horas, no se requirió transfusión, el paciente toleró la vía oral a las doce horas siguientes, y fue dado de alta en el primer día postoperatorio. Se retira dren al cuarto día y la sonda uretral a las dos semanas. El paciente recobró continencia al final del primer mes sin requerimiento de pañal, sin erecciones espontáneas y con prueba de alprostadil positiva. La patología reportó adenocarcinoma de próstata Gleason $3+3$ grado 6 que comprometía ambos lóbulos, con bordes de sección y vesículas seminales positivos, y linfadenectomía ilioobturadora bilateral negativa. Se cataloga como T3bNoMo. El PSA postoperatorio fue de 2,98 y en la actualidad se encuentra con terapia coadyuvante por sus márgenes positivos.

\section{Discusión}

Como toda técnica nueva, la prostatectomía radical laparoscópica está sometida a una serie de cambios con el propósito de perfeccionarla. El procedimiento descrito inicialmente por Vallancien y Guillonneau se ha ido depurando, haciéndolo más accesible y en la actualidad se realiza tanto de forma transperitoneal como extraperitoneal. Aunque en esta oportunidad no se preservaron las bandeletas neurovasculares por la extensión del tumor, la conservación de éstas como técnica laparoscópica es mucho más factible que en la cirugía abierta por la magnificación del campo operatorio. Entre los cambios efectuados se encuentra la forma de realización de la anastomosis, la cual se hace en sutura continua, disminuyendo así el número de días requeridos de cateterización urinaria. El tiempo quirúrgico ha disminuido en forma gradual. El grupo de Cleveland liderado por Inderbier Gill reportó un promedio de tiempo operatorio de 5,5 horas en sus primeros 44 casos, el cual fue decreciendo a medida que avanzó en la curva de aprendizaje; el grupo del Montsouris del de Vallancien y Guillonneau informan un tiempo operatorio de 170 minutos.

Una ventaja adicional de la cirugía laparoscópica es la disminución del sangrado en relación con la cirugía abierta, debida a la excelente visibilidad del complejo dorsal y al efecto de taponamiento que realiza la pre- sión del neumoperitoneo durante la cirugía. El sangrado es el principal enemigo de la laparoscopia al alterar la visión, por lo cual debe evitarse durante todo el procedimiento. La variación de la hemoglobina preoperatoria y a las 24 horas en el grupo de la Floresta fue de 1,6 $\mathrm{mg} / \mathrm{dl}$. Las tasas de márgenes positivos son comparables con la cirugía abierta. El grupo de Creteil con pT3 como nuestro paciente es del $48 \%$ y en los pT 2 varía entre el 16 y el $25 \%$.

Los índices de continencia y de estrechez de la anastomosis son comparables en las diferentes series. Esperamos mantenerla en el 95\% reportado para nuestras series abiertas. Las bajas tasas de morbilidad en la cirugía laparoscópica permiten a los pacientes la pronta recuperación, con lo que se logra una estadía hospitalaria más corta que en el procedimiento abierto. El promedio de estancia reportado por Steinbergh y Hill fue de 1,2 días.

En conclusión, la prostatectomía radical laparoscópica es un procedimiento novedoso que está mostrando beneficios, en comparación con la prostatectomía radical abierta, entre los cuales se encuentran la posibilidad de magnificar el campo para preservar las bandeletas nerviosas, el menor sangrado, la disminución de la estancia hospitalaria, la recuperación rápida con menor requerimiento de analgésicos y la incorporación laboral temprana con menor incapacidad.

\section{Agradecimientos}

A la doctora Laima Didziulis, Directora del Hospital de San José, por su constante apoyo en el desarrollo de las nuevas técnicas.

\section{Lecturas recomendadas}

1. Guillonneau B, Vallancien G, Olsson CA. Laparoscopicradical prostatectomy: The montsouris technique. The Journal of Urology 2000; 163:1643-1649

2. Valleancien G, Guillonneau B, Fournier G, et al. Prostatectomía radical laparoscópica. Manual de técnica quirúrgica, Primera Edición, París Francia. Les Editions 21-2002, 1-126.

3. Parsons JK, Jarrett TJ, Chow GK, Kavoussi LR. The effect of previous abdominal surgery on urological laparoscopy. The Journal of Urology Dec 2002; 168(6): 2387-2390. 
4. Stolzenburg JU, Do M, Rabenalt R, et al. Endoscopic extraperitoneal radical prostatectomy: initial experience after 70 procedures. The Journal of Urology. Jun 2003; 169(6): 2066-2071.

5. Guillonneau B, Vallancien G. Laparoscopic radical prostatectomy: the Montsouris experience. The Journal of Urology 2000; 163:418.

6. Katz R, Salomon L, Hoznek A, et al. Patient reported sexual function following laparoscopic radical prostatectomy. The Journal of Urology. Nov 2002; 168(5): 2078-82.

7. Nadu A, Salomon L, Hoznek A, et al. Early removal of the catheter after laparoscopic radical prostatectomy. The Journal of Urology 2001; 166:1662-64.

8. Rassweiler J, Seemann O, Schulze M, et al. Laparoscopic versus open radical prostatectomy: a comparative study at a single institution. The Journal of Urology May 2003; 169(5): 1689-93.

9. Guillonneau B, Rozet F, Cathelineau X, et al. Perioperative complications of laparoscopic radical prostatectomy: the Montsouris 3-year experience. The Journal of Urology. 2002; 167:51-56.
10. Guillonneau B, El-Fettouh H, Baumert H, et al. Laparoscopic radical prostatectomy: oncological evaluation after $1.000 \mathrm{ca}-$ ses at Montsouris institute. The Journal of Urology. Apr 2003; 169(4): 1261-66.

11. Katz R, Salomon L, Hoznek A, et al. Positive surgical margins in laparoscopic radical prostatectomy: the impact of apical dissection, bladder neck remodeling and nerve preservation. The Journal of Urology. Jun 2003 169(6): 2049-52.

12. Rassweiler J, Tsivian, A, Ravi Kumar AV, et al. Oncological safety of laparoscopic durgery for urological malignancy: experience with more than 1.000 operations. The Journal of Urology. Jun 2003; 169(6): 2072-75.

13. Soulie M, Seguin P, Richeux L, et al. Urological complications of laparoscopic surgery: experience with 350 procedures at a single center. The Journal of Urology. 2001;165:1960-63.

14. Vallancien G, Cathelineau X, Baumert, $\mathrm{H}$, et al. Complications of transperitoneal laparoscopic surgery in urology: review of 1.311 procedures at a single center. The Journal of Urology. 2002; 168:23-26.

15. Hara I, Kawabata G, Miyake H, et al. Comparison of quality of life following laparoscopic and open prostatectomy for prostate cancer. The Journal of Urology. Jun 2003; 169(6): 2045-48. 